MILITARY TECHNICAL COLLEGE CAIRO - EGYPT $1^{\text {st }}$ INTERNATIONAL CONF. ON ELECTRICAL RNGINEERING

\title{
PERFORMANCE OF A LOW BIAS DF ESTIMATOR IN WIDE BAND COHERENT SOURCES ENVIRONMENT
}

\author{
M. M. Mokhtar and S. S. Besar ${ }^{\circ}$
}

\begin{abstract}
The estimation of radio activities location is a main task of radio reconnaissance systems. The performance measures of the estimators of these systems are usually the resolution threshold, bias, and variance of the estimated directions. Recently, a low bias estimator, named $\mathrm{D}_{\mathrm{r}}$, is proposed for applications in narrow band incoherent sources environment. In this paper, some attractive features of the $D_{r}$ in a practical environment of wide band coherent sources are introduced. It also presents a process, based on the $\mathrm{D}_{\mathrm{r}}$ for attaining a low resolution threshold with a low level of both bias and variance.
\end{abstract}

\section{Key Words}

Direction Finding, DSP

\section{Introduction}

In Direction Of Arrivals (DOA's) estimation problems, the variance, bias, and resolution threshold are the measures of the estimator performance. Most of the recent estimators are based on the Multiple Signal Classification (MUSIC) estimator which has been originally developed for narrow band incoherent sources environment problems. Theoretical study of the MUSIC estimator shows that it possess good asymptotic variance, but with high resolution threshold and driven bias. In the last few years, an attention has been given to reduce the estimation bias and/or resolution threshold. An improvement of both, in some works, comes on the account of the variance of the estimated angles. Recently, two MUSIC like estimators - the weighted norm MUSIC and $D_{r}$ estimators have been proposed [1]. The analytical and simulation studies of these estimators in narrow band incoherent sources environment show that they have the same asymptotic variance of the MUSIC but with lower bias and resolution limit. In the environment of coherent sources, one has to expect a

* Egyptian Armed Forces 
performance much less than that has been estimated. The most widely used techniques for alleviating this problem are the spatially smoothing $[2,3]$, and focusing techniques $[4,5]$. Normally, one of these techniques is combined with a selected DOA's estimator to resolve the signal impinging on an array of sensors.

This paper presents a comparison between the MUSIC in conjunction with the Rotational Signal Subspace (RSS) focusing techniques [5], and the $\mathrm{D}_{\mathrm{r}}$ estimators using a uniform linear array. The results show also that a better performance than both can be attained when the $\mathrm{D}_{\mathrm{r}}$ estimator is combined with the RSS technique. Different array geometries can also be employed using the transformation techniques as described in $[6,7]$.

\section{Model and Basic Assuraptions}

Let $\mathbf{R}$ is the sample covarianc: matrix based on $\mathrm{N}$ independent snapshots of the received signal from uniform linear array of $\mathbf{L}$ elements. The geometry of the array is described by the steering vector $v(\vartheta)$ in the elevation as

$$
\nu(\vartheta)=\left[1, e^{i 2 \pi f D \cos (\xi) / c}, \ldots \ldots, e^{i 2 \pi f D(L-1) \cos (\vartheta) / c}\right]
$$

where $\mathrm{D}$ is the interelement spacing, $\mathrm{c}$ is the wave velocity in space, $\mathrm{f}$ is the frequency of the received signal, and $\vartheta$ is the angle between the direction of arrival and the array axis. Assume that $\lambda_{\nu_{i}}$ and $e_{i}$ are the $i^{\text {th }}$ eigenvalue and normalized eigenvector of R. If the first $\mathbf{d}$ eigenvalues are distinct and greater than the variance of an additive white (Gaussian noise $\sigma_{n}^{2}$; i.e.

$$
\lambda_{1}>\lambda_{2}>\ldots \ldots>\lambda_{\mathrm{d}}>\lambda_{\mathrm{d}+1}=\ldots \ldots=\lambda_{\mathrm{L}}=\sigma_{\mathrm{n}}^{2},
$$

the signal subspace $S_{s}$ and noise (null) subspace $S_{n}$ are defined as

$$
\begin{aligned}
& S_{s}=\operatorname{span}\left\{e_{1}, e_{2}, \ldots . e_{d}\right\} \\
& S_{n}=\operatorname{span}\left\{e_{d+1}, \ldots . . e_{L}\right\}
\end{aligned}
$$

In the fol'.owing, the definitions

$$
\begin{aligned}
& E_{s}=\left[e_{1}, e_{2}, \ldots . e_{d}\right], \\
& E_{n}=\left[e_{d+1}, \ldots \ldots e_{L}\right], \\
& P_{s}=E_{s} E_{s}^{H}, \text { and } \\
& P_{n}=E_{n} E_{n}^{H}
\end{aligned}
$$

are used in the expression of the MUSIC and $\mathrm{D}_{\mathrm{r}}$ estimators, where $\left[{ }^{*}\right]^{\mathrm{H}}$ denotes the hermitance transpose of the vector [*]. The eigenanalysis of $\mathrm{R}$ is carried at $\mathrm{K}$ discrete frequencies uniformly distributed over the bandwidth of interest. 


\section{The MUSIC Estimator}

The MUSIC estirnator is defined, for wide band sources, as [8]

$$
P(\vartheta)=\frac{1}{\frac{1}{K} \sum_{k=1}^{K}\left[\left\|\left(I-E_{s} E_{s}^{H}\right) v(\vartheta)\right\|^{2}\right]_{f_{k}}}
$$

\section{The $D_{\mathrm{r}}$ Estimator}

The $D_{r}$ estimator a class of the MUSIC, is formulated as a minimization of the Euslidean distance between two vectors in the signal subspace [1]. Let the set $E_{s}$ is decomposed into $E_{s}^{(1)}$ and $E_{s}^{(2)}$, and define

$$
\begin{aligned}
& f^{(1)}(\vartheta)=\left(I-E_{s}^{(1)} E_{s}^{(1) H}\right) v(\vartheta) \\
& f^{(2)}(\vartheta)=E_{s}^{(2)} E_{s}^{(2) H} v(\vartheta)
\end{aligned}
$$

The $\mathrm{D}_{\mathrm{r}}$ estimator is defined by

$$
P^{\prime}(\vartheta)=\frac{1}{\frac{1}{K} \sum_{k=1}^{k} \min \cdot\left[\left\|f^{(1)}(\vartheta)\right\|^{r(\vartheta)}-\left\|f^{(2)}(\vartheta)\right\|^{r(\vartheta)}\right]_{f_{k}}}
$$

where

$$
r(\vartheta)=\frac{1}{\log \left\|f^{(2)}(\vartheta)\right\|}
$$

\section{The RSS Estimator}

The general techr ique of focusing is based on averaging the covariance matrices at the selected $\mathrm{K}$ discre e frequencies in the form

$$
\bar{R}=\sum_{k=1}^{K} T_{k} R_{k} T_{k}^{H}
$$

where $T_{k}$ and $R_{k}$ cre the focusing and covariance matrices at the frequency $f_{k}$, respectively. The eigenvectors of the steered covariance matrix is used for estimating the directions of radio activities. The form of the estimator is similar to that of the MUSIC for narrc w band sources. The $T_{k}$ is defined as a rotational matrix of the steering vector $v(\vartheta)$ at $f_{k}$ to that at the center frequency $f_{o}$. The detail of $T_{k}$ generation is givın in [5]. 


\section{Simulation Results}

Let the number of omni-directional sensors of a uniform linear array is 7 with interelements spacing is 0.5 the wavelength $\lambda$ of the center frequency $f_{o}$. Consider a problem of two closely space 1 equipower wideband coherent sources with band width $0.125 f_{o}$. The sources direction are defined by the center angle $\vartheta_{o}$ between the sources measured from the array axis, and the angle of separation $\Delta$ normalized to the BeamWidth (BW) of the array. L'et $\Delta$ equals $0.2 \mathrm{BW}$, and select the width of the focusing domain to $1.25 \Delta$; thes optimum focusing width as proposed in [7]. Figure 1 shows both the bias and variance, normalized to $\mathrm{BW}$, of the estimated directions versus SNR in $\mathrm{dB}$ using the RSS, $\mathrm{D}_{\mathrm{r}}$, and $\mathrm{D}_{\mathrm{r}}$ - RSS estimators in the two cases of $\vartheta_{o}=90^{\circ}$ and $60^{\circ}$. Figures $1 . \mathrm{a}$ and $\mathrm{b}$, for $\vartheta_{o}=90^{\circ}$, show that the resolution threshold of the RSS is $10 \mathrm{~dB}$ and higher than that of the $\mathrm{D}_{\mathrm{r}}$, but with lower bias and variance. This result gives rise to an idea of combining the $D_{r}$ with RSS estimators for attaining the lower resolution threshold of the $\mathrm{D}_{\mathrm{r}}$, with a lower level of both the bias and variance of the RSS. In this approach, which is named $D_{r}-R S S$, the $D_{r}$ is applied to a single focused covariance matrix of the RSS. The dash-dot curves in Figs. 1.a and b show that the $\mathrm{D}_{\mathrm{r}}-\mathrm{RSS}$ has a performance much better than that of the $\mathrm{D}_{\mathrm{r}}$ and RSS. In the above problem with $\vartheta_{0}=60^{\circ}$, the performance of the three estimators are less than those in case of $\vartheta_{o}=90^{\circ}$; compare Figs. 1.a with 1.c, and 1.b with 1.d. Figures 1.c and d show also that the performance of the $\mathrm{D}_{\mathrm{r}}-\mathrm{RSS}$ is better than the others.

The main difficulties in the application of the RSS estimator are the preliminary estimation of both the $\vartheta_{o}$ and the width of the focusing domain. In the above results, the angle $\vartheta_{o}$ is chosen in the direction of a single peak in the MUSIC estimated spectrum, while the focusing width is increased in steps of length .01BW until minimum levels of both the bias and variance are attained. Indeed, this is a complicated process to be applied in practice. Therefore the following process, named $\mathrm{D}_{\mathrm{r}} / \mathrm{RSS}$, is suggested to simplify the application of the RSS. Consider the above problem with $\vartheta_{o}=90^{\circ}$, and $\mathrm{SNR}=5 \mathrm{~dB}$. The estimated spectrum of the MUSIC estimator (Fig.2.a) shows an unresolved spectrum with a single peak in the direction of $\vartheta_{o}=90^{\circ}$. The arrows in the Figure mark the real directions of the sources. This single peak may be interpreted as a single source in the peak direction, or an unknown number of coherent sources in unknown directions. Since the $\mathrm{D}_{\mathrm{r}}$ estimator has a low resolution threshold, it can be used to resolve this peak, and hence preliminary estimate the sources location. Fig.2.b shows that the estimated spectrum of the $\mathrm{D}_{\mathrm{r}}$ comprises two distinct peaks in the directions of $\vartheta_{o}=90^{\circ}$ and $\vartheta_{o}=90^{\circ}$. The next step is applying the RSS using two groups of focusing in $\vartheta_{1}$ and $\vartheta_{2}$ directions with a domain width of focusing for each is $\leq\left|\vartheta_{1}-\vartheta_{2}\right|$. Figure 2.c shows that the peak of 
Proceeding of the $1^{\text {st }}$ ICEENG conference, 24-26 March, 1998

the spectrum become very close to the real directions. For further retinment, the RSS can be applied once again in the new directions, but with reduced do main of focusing.

\section{Conclusion}

The $D_{r}$ estimator, originally developed for narrcw band incoherent s ources, has a low resolution threshold when applied in the environment of wide band coherent sources. This feature can be used to circumvent the difficulties in the applicat ion of the Rotational Signal Subspace (RSS) focusing technique. These difficulties are the preliminary estimation of both the sources direction and the domain 'width of focusing. The numerical results show that the conjunction of the $\mathrm{D}_{\mathrm{r}}$ and RSS estimators in a process named $\mathrm{D}_{\mathrm{r}} / \mathrm{RSS}$ may yields a much better pertormance than each.

\section{References}

[1] Xu. Wenyuan and M.Kaveh,"Design of two MUSIC-like estimat.srs based on bias minimization", IEEE Tran. Signal Process., Vol.44, No.9, Sept. 1996, pp 2284-2299. [2] Shan, J., Wax, M., and Kailath, T.,"On spatial smoothing for dire ztion-of-arrival estimation of coherent signals", IEEE Trans. Acoustic, speech, and signal processing, Vol.33, No.4, Aug. 1985, PP.806-811.

[3] Williams, R.T., Prasad, S., Mahalancbis, A.K., and Sibul, L.H.,"in improved spatial smoothing technique for bearing estimation in a multipath en 'ironment", IElEE Trans. Acoustic, speech, and signal processing, Vol.36, No.4, Apr. 1988, PP.425-432. [4] Wang, H., and Kaveh, M.,"Coherent signal subspace processing tor the detection and estimation of angle of arrival wide-band sources", IEEE Trans. Acoustic, speech, and signal processing, Vol.33, No.4, Aug. 1985, PP.822-831.

[5]Hung, H., and Kaveh, M.,'Focusing matrices for coherent signal subsprace processing", IEEE Trans. Acoustic, speech, and signal processing, Vol.36, No.8, Aug. 1988, PP.1272-1281.

[6] Doron, M.A., Doron, E., and Weiss A.J.," Coherent wide-band processing for arbitrary array geometry", IEEE Trans. Signal Process., Vol.41, Jan. 1993, PP.414417.

[7] M. Mokhtar, M. Abdel kader, and M. Kassem,'Direction of arrival estimation techniques using uniform circular array", Proc. of $13^{\text {th }}$ Nat. Radio Sc ence Conf., Cairo, Egypt, 1996, PP.1-8(B2).

[8] Wax, M., Shan, T., and Kailath, T.,"Spatio-temporal spectral analysis by eigenstructure methods", IEEE Acoustic, speech, and signal processi.ng, Vol.32, No.4, Aug. 1984, PP.817-827. 
Proceeding of the $1^{\text {st }}$ ICEENG conference, 24-2.6 March, 1998.

SP. 159
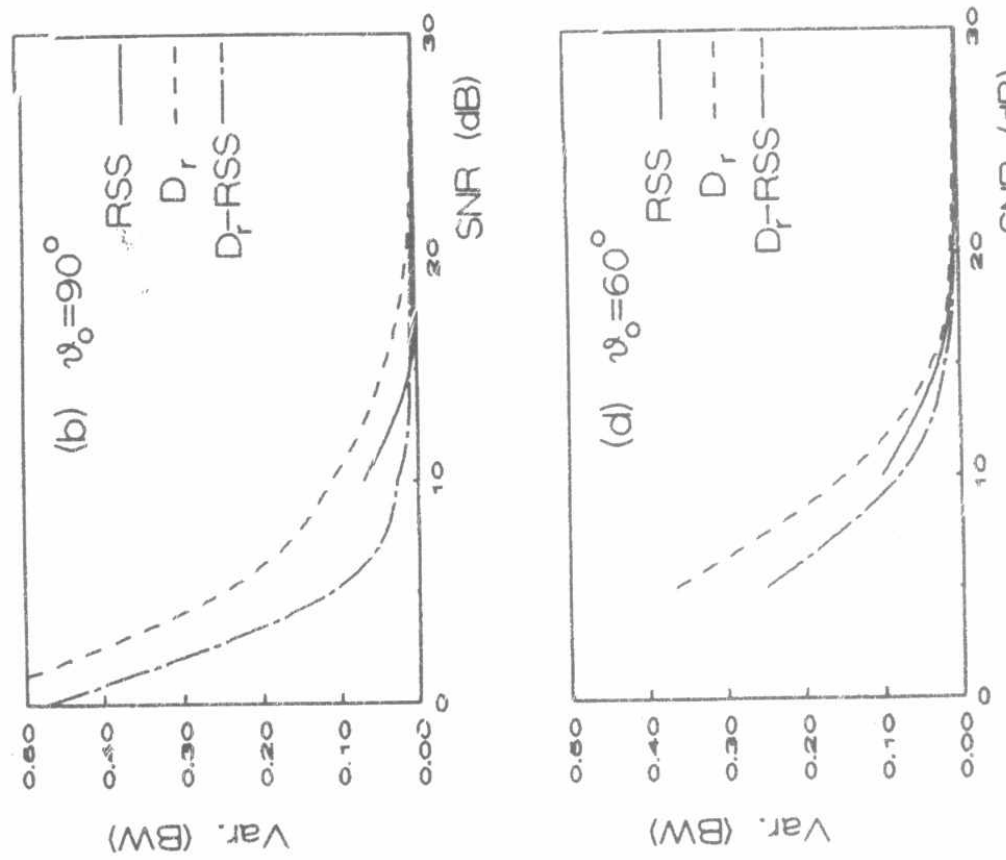

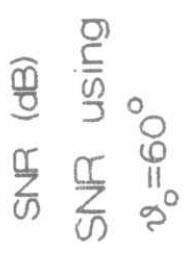
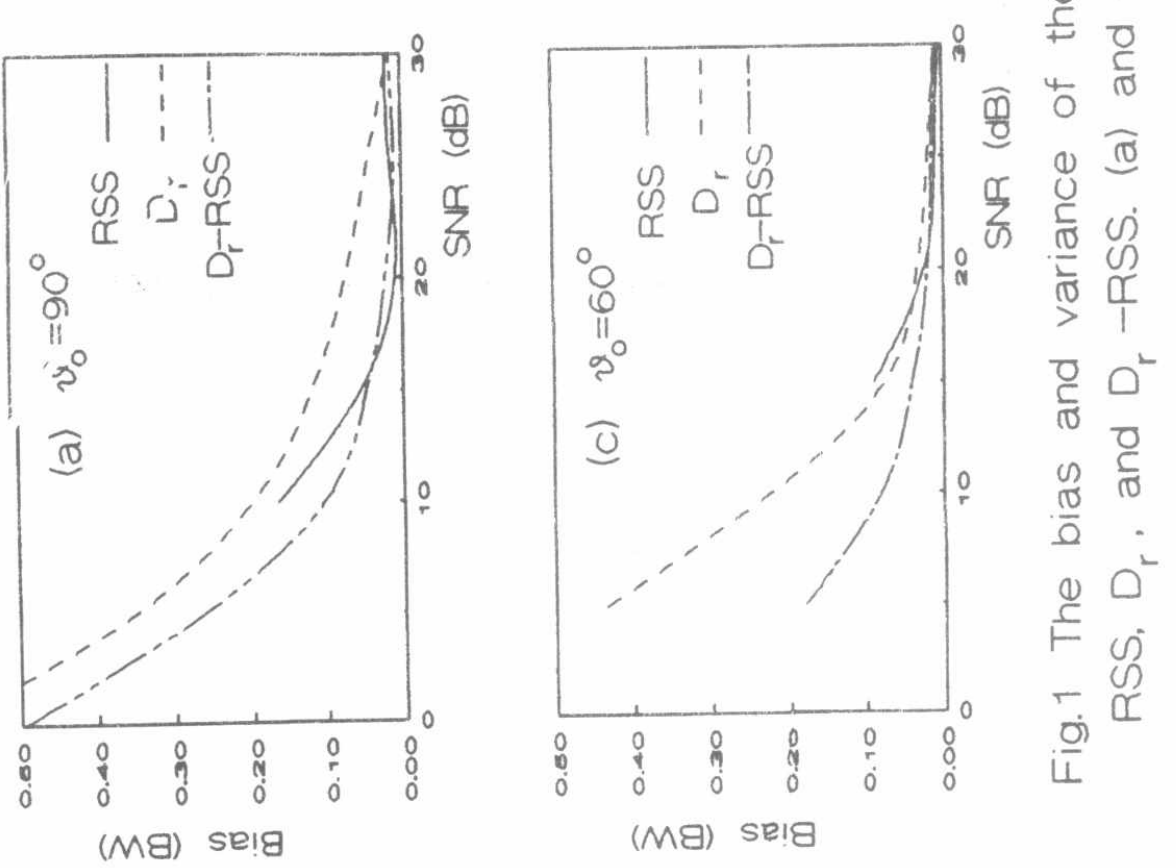
Proceeding of the $1^{\text {st }}$ ICEENG conference, 24-26 March, 1998.
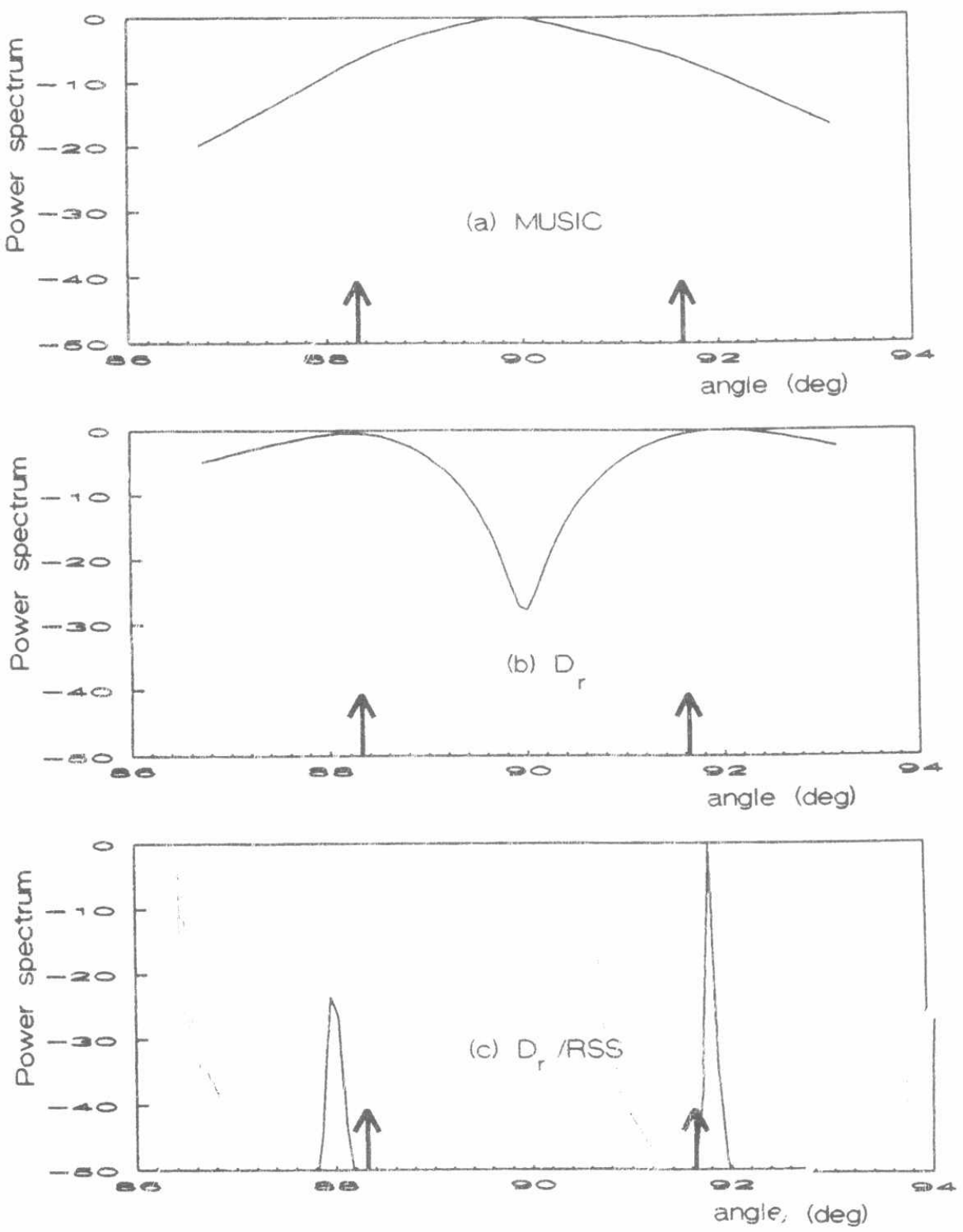

Fig.2 The estimated power spectrum of two coherent sources.
(a) MUSIC
(b) Dr
(c) $D_{r} / R r j s$ 